\title{
COMPLETE REGENERATION OF LARGE BONE DEFECTS IN RATS WITH COMMER CIALLY AVAILABLE FIBRIN LOADED WITH BMP-2
}

\author{
M. Koolen ${ }^{1, *}$, A. Longoni ${ }^{2,3}$, J. van der Stok ${ }^{4}$, O. Van der Jagt ${ }^{5}$, D. Gawlitta ${ }^{2,3}$ and H. Weinans ${ }^{1,6}$ \\ ${ }^{1}$ Department of Orthopaedics, University Medical Centre Utrecht, Utrecht University, \\ Utrecht, the Netherlands \\ ${ }^{2}$ Department of Oral and Maxillofacial Surgery and Special Dental Care, University Medical Centre \\ Utrecht, Utrecht University, Utrecht, the Netherlands \\ ${ }^{3}$ Regenerative Medicine Centre Utrecht, Utrecht, the Netherlands \\ ${ }^{4}$ Department of Orthopaedics, Erasmus University Medical Centre Rotterdam, \\ Rotterdam, the Netherlands \\ ${ }^{5}$ Department of Orthopaedics, Elisabeth-TweeSteden Hospital, Tilburg, the Netherlands \\ ${ }^{6}$ Department of Rheumatology and Clinical Immunology, University Medical Centre Utrecht, \\ Utrecht, the Netherlands
}

\begin{abstract}
This study aimed at investigating in vitro and in vivo the efficiency of commercially available fibrin as a carrier for controlled and sustained bone morphogenetic protein-2 (BMP-2) release to induce bone formation and reduce the side effects of its use. In vitro release and activity of low-dose recombinant human BMP-2 (rhBMP-2) $(37.5 \mu \mathrm{g} / \mathrm{mL})$ embedded in commercially available fibrin were evaluated and, subsequently, criticalsize femur defects in rats were grafted to study bone regeneration and vascularisation by micro-computed tomography $(\mu \mathrm{CT})$ and histology. In vitro experiments showed a sustained BMP-2 release with a high BMP activity remaining after $28 \mathrm{~d}$. In vivo, fibrin loaded with BMP-2 showed an extremely fast bone healing, with a large amount of new bone formation throughout the entire defect in the first 4 weeks and complete cortical repair and fusion after 8 weeks, with no ectopic bone formation. In contrast, the control fibrin group did not fuse after 12 weeks. Vascularisation was similar in both groups at 4 and 12 weeks after implantation. In conclusion, commercially available fibrin is a very efficient carrier for rhBMP-2 to graft critical-size cortical bone defects and might be a more optimal delivery vehicle for BMP-2-induced bone regeneration than currently available collagen sponges.
\end{abstract}

Keywords: Preclinical studies, non-union, bone morphogenetic proteins, bioengineering, implants, injury/ fracture healing.

*Address for correspondence: M.K.E. Koolen, Department of Orthopaedics, UMC Utrecht, G.05.228, P.O. Box 85500, 3508 GA Utrecht, the Netherlands.

Telephone number: +31 887559003 Email: m.k.e.koolen@umcutrecht.nl

Copyright policy: This article is distributed in accordance with Creative Commons Attribution Licence (http://creativecommons.org/licenses/by-sa/4.0/).

\section{Introduction}

Bone is a diverse tissue, which can regulate, adapt and regenerate itself after damage. However, the extent of regeneration is limited, especially when infectious or non-vital tissue is present or when bone defects are too large to be bridged, e.g. after major trauma, tumour resection or revision arthroplasty (Giannoudis et al., 2005). Insufficient bone regeneration leads to nonunions or large persistent bone defects and their repair remains a major issue in orthopaedic surgery (Einhorn, 1995). Natural grafts (e.g. autografts and allografts) have certain drawbacks, both with respect to quantity (availability of satisfactory grafts) as well as quality (donor site morbidity, graft rejection and disease transmission) (Dimitriou et al., 2011). Therefore, there is considerable interest in novel bone regeneration methods.

Three main elements supporting bone regeneration are well-known, e.g. cell sources, scaffolds and tissueinducing factors (signalling factors) or so-called bioactive factors. These bioactive factors, used in combination or alone, are a very potent element to treat patients with irreversible bone defects. The most 
well-known bioactive factors for bone regeneration are recombinant human bone morphogenetic proteins (rhBMPs). BMPs are naturally produced during fracture repair and fulfil multiple important roles during skeletal formation (Dimitriou et al., 2005). For more than two decades, two types of rhBMP have been clinically used to repair bone defects or treat non-unions: rhBMP-2 and rhBMP-7.

Both rhBMP-2 and rhBMP-7 are clinically effective and received Food and Drug Administration (FDA) approval to be used in fracture repair of tibia and spinal fusion (Garrison et al., 2007; Ong et al., 2010). However, with their increased use, a growing and well-documented side effect profile has emerged, including local swelling, ectopic bone formation, bone resorption, urogenital events and wound complications (James et al., 2016). These disadvantages are believed to be related to the high dosages which are necessary to achieve bone formation (Carragee et al., 2011; Termaat et al., 2005). These adverse events associated with rhBMP-2 use in spine fusion range from 10 to $50 \%$ and this risk is probably equivalent to or greater than that of iliac crest bone graft harvesting (Carragee et al., 2011). This led to a negative tendency towards rhBMPs' use and trials are now aimed toward elucidating the optimal rhBMP-2 dosage (James et al., 2016). To get the optimal dose and timing of rhBMP-2 activity, the need for a proper delivery system has emerged (Termaat et al., 2005). The current FDA-approved formulation of rhBMP-2 uses a release system, where BMP-2 is placed in an absorbable collagen sponge. However, this collagen sponge quickly releases BMP2 and is, therefore, loaded with a high dose of BMP2 (Benglis et al., 2008). A suitable delivery system should enable a controlled and sustained release of rhBMPs, thereby avoiding the need for a high dose, which is likely related to the aforementioned side effects (Begam et al., 2017).

An ideal carrier material should closely mimic the natural environment during bone regeneration, thereby functioning as an extension of the body's own repair capacities. One of the first steps in natural bone regeneration is the formation of a blood clot. This clot is derived from fibrinogen, which forms a nano-/micro-fibrillar meshwork under the enzymatic action of thrombin (Rajangam and An, 2013). It provides a favourable environment for cellular attachment and proliferation and for cytokines, including BMPs. Clinically, fibrinogen is already used as fibrin glue (Noori et al., 2017). These fibrin glues purposely use a supraphysiological concentration of fibrinogen (50-100 mg/mL) to form a dense meshwork that functions as a haemostatic agent. However, lower fibrinogen concentrations increase the permeability of the meshwork, which seems favourable for rapid cell invasion, remodelling and replacement by transplanted or host cells (Janmey et al., 2009). Previous experiments using specifically designed high-molecular-weight fibrin gels, with low physiological concentrations $(5 \mathrm{mg} / \mathrm{mL})$ and mixed with low-dose BMP-2, have been shown to be an excellent delivery system to enhance bone regeneration of large bone defects (van der Stok et al., 2015).

The aim of the present study was to determine whether commercially available fibrin as a carrier for low-dose rhBMP-2 was also effective in inducing bone formation in a large rat cortical femoral defect, thereby paving the way for possible future clinical applications in trauma and orthopaedic surgery.

\section{Materials and Methods}

\section{Study design}

To evaluate the performance of a fibrin hydrogel as a BMP-2 carrier in vitro, the release profile of the protein was analysed for $28 \mathrm{~d}$. Further, to assess whether the released BMP-2 was still active, its capability of inducing an increase in alkaline phosphatase (ALP) activity was tested in ATDC5 cells. Finally, the construct degradation was monitored over time. To check whether the concentration and release profile determined in vitro were suitable for in vivo applications, BMP-2-loaded constructs were implanted in a critical-size femur defect in rats. A construct composed of only fibrin was used as control. Fibrin (fibrin) and BMP-2 embedded in fibrin (fibrin-BMP) groups were compared for their ability to promote bone formation and vascularisation. Micro-computed tomography $(\mu \mathrm{CT})$ and histology were used for result evaluation.

\section{Fibrin construct preparation}

Fibrin gels were prepared in a sterile environment using cuboid shaped custom-made moulds $(6 \times 3.5 \times 3.5 \mathrm{~mm})$ having the same size as the critical-size femur defect. Clinically applicable human fibrinogen (Tissucol, Baxter) was diluted in sterile phosphate buffered saline (PBS) to a final concentration of $22.5 \mathrm{mg} / \mathrm{mL}$ and clotted for $30 \mathrm{~min}$ at room temperature with $2.5 \mathrm{IU} / \mathrm{mL}$ thrombin (Global Siemens Healthcare, Erlangen, Germany) dissolved in a $20 \mathrm{mM}$ calcium chloride solution (total volume $80 \mu \mathrm{L})$. Constructs with fibrin and BMP-2 were prepared by adding $3 \mu \mathrm{g}$ of rhBMP-2 (InductOS, Wyeth/Pfizer, New York, NY, USA) to this total volume, resulting in a final concentration of $3 \mu \mathrm{g} B M P$ per $0.08 \mathrm{~mL}$, i.e. $37.5 \mu \mathrm{g} / \mathrm{mL}$. This concentration was chosen based on previous studies showing that 2-5 $\mu \mathrm{g}$ BMP-2 is sufficient to stimulate bone regeneration in a $6 \mathrm{~mm}$ segmental femur bone defect in rats (Angle et al., 2012; Bessa et al., 2010; Boerckel et al., 2011; van der Stok et al., 2015; Wei et al., 2007; Zara et al., 2011). Constructs were prepared one by one to prevent premature setting of the gels during handling and an inhomogeneous dispersion of BMP-2.

Constructs were prepared on the day before surgery and, to prevent dehydration, they were incubated at $37{ }^{\circ} \mathrm{C}$ overnight in $1 \mathrm{~mL}$ of medium consisting of high-glucose Dulbecco's modified Eagle 
medium (DMEM; 31966; Gibco) supplemented with $1 \%$ ITS+ premix (354352; BD Biosciences), $10^{-7} \mathrm{M}$ dexamethasone (D8893; Sigma-Aldrich), $0.2 \mathrm{mM}$ L-ascorbic acid 2-phosphate (A8960; Sigma-Aldrich), $50 \mu \mathrm{g} / \mathrm{mL}$ gentamicin (17-518Z; Lonza) $1.5 \mu \mathrm{g} / \mathrm{mL}$ fungizone (15290-026; Thermo Fisher Scientific) and $40 \mu \mathrm{g} / \mathrm{mL}$ L-proline (P-5607; Sigma-Aldrich). This basal chondrogenic medium was used because natural fracture healing first generates cartilage.

For in vitro experiments, gels were cultured in $1 \mathrm{~mL}$ of DMEM/F12 (1: 1) plus GlutaMAX (31331093; Thermo Fisher Scientific) supplemented with $10 \%$ heat-inactivated foetal bovine serum (FBS) (RWG35912; HyClone GE Healthcare), 100 U/ $\mathrm{mL}$ penicillin and $100 \mathrm{mg} / \mathrm{mL}$ streptomycin (15140; Thermo Fisher Scientific). Three groups were compared during in vitro analysis: fibrin (fibrin), BMP-2 embedded in fibrin (fibrin-BMP) and BMP2 embedded in fibrin and maintained in medium containing plasmin $(0.025 \mathrm{U} / \mathrm{mL}, \mathrm{P} 1867$; SigmaAldrich) (fibrin-BMP-plasmin). Plasmin was added to the medium to promote a faster degradation of the fibrin gels, as plasmin in vivo cuts a fibrin mesh at various places during fibrinolysis (Bannish et al., 2017; Schmoekel et al., 2005) and, therefore, the plasmin group might provide a more realistic representation of the in vivo situation. Construct length was measured using an Olympus BX51 microscope (Olympus DP70 camera, Olympus) to visualise degradation of the constructs over time.

\section{In vitro release of $\mathrm{BMP}-2$}

BMP-2 release over time was evaluated using a BMP-2 enzyme-linked immunosorbent assay (ELISA) (900-M255; PeproTech) according to manufacturer's instructions. Since no BMP-2 was loaded in the fibrin group, only the fibrin-BMP and the fibrin-BMPplasmin groups were analysed for BMP-2 content. Medium collected from the constructs $(n=3)$ at day $0,1,2,5,10,16,21$ and 28 was stored at $-80^{\circ} \mathrm{C}$ until ELISA could be performed. Fresh, non-cultured medium was used as a negative control. The plate was read at different time points $(5,10,15$ and $20 \mathrm{~min})$ at $405 \mathrm{~nm}$ with wavelength correction set at $650 \mathrm{~nm}$.

\section{In vitro BMP-2 activity}

To assess if the fibrin crosslinking process could have affected BMP-2 activity, its ability to enhance ALP activity was evaluated in ATDC5 cells. Using two hydrogels for each group, fibrin, fibrin-BMP and fibrin-BMP-plasmin were incubated in medium for 10 or 28 consecutive days at $37^{\circ} \mathrm{C}$ under humidified conditions and $5 \% \mathrm{CO}_{2}$. Fibrin and medium incubated for an equivalent number of days were taken along as negative controls.

$3 \mathrm{~d}$ before the time points ( 10 or $28 \mathrm{~d}$ ), 6,000 cells/ well were seeded in a 96 well plate. ATDC5 cells were cultured at $37^{\circ} \mathrm{C}$ under humidified conditions and $5 \% \mathrm{CO}_{2}$ in DMEM/F12 (1: 1) plus GlutaMAX (31331093; Thermo Fisher Scientific) supplemented with $10 \%$ heat-inactivated FBS (RWG35912; HyClone
GE Healthcare), $100 \mathrm{U} / \mathrm{mL}$ penicillin and $100 \mathrm{mg} /$ mL streptomycin (15140; Thermo Fisher Scientific). After $3 \mathrm{~d}$, the medium in which the constructs of the three groups were placed was collected and added to the ATDC5 cells ( $n=3$ per hydrogel) at different dilutions (undiluted and $10 \times$-diluted). Then, cells were incubated for another $3 \mathrm{~d}$, after which ALP activity was evaluated.

Briefly, after washing with PBS, ATDC5 cells were lysed with $0.5 \%$ Triton-X 100 in PBS for $30 \mathrm{~min}$. Of each sample, $25 \mu \mathrm{L}$ were incubated in a flat transparent bottom 96 well plate with $50 \mu \mathrm{L}$ para-nitrophenylphosphate (pNPP) (N7653; SigmaAldrich). ALP enzyme (P4978; Sigma-Aldrich) with known U/mL was used to obtain a standard curve. A kinetic reading was performed every $2 \mathrm{~min}$ for $30 \mathrm{~min}$ at $405 \mathrm{~nm}$ with a correction at $655 \mathrm{~nm}$.

To correct for the number of cells present in each well, DNA was quantified using Quant-iT ${ }^{\mathrm{TM}}$ PicoGreen $^{\mathrm{TM}}$ dsDNA Assay Kit (P7589; Thermo Fisher Scientific) according to the manufacturer's instructions.

\section{Animals}

18 male Wistar rats (Charles River) were housed in pairs under strict supervision in the animal facility of the University Medical Centre Utrecht. Animals received standard food pellets and water ad libitum and were kept under climate-controlled conditions $\left(21{ }^{\circ} \mathrm{C} ; 12 \mathrm{~h}\right.$ light/12 $\mathrm{h}$ darkness). At the age of 16 weeks, after at least $7 \mathrm{~d}$ of acclimatisation in the animal facility, a $6 \mathrm{~mm}$ critical-size segmental bone defect was created (van der Stok et al., 2013a), as briefly described below. Two experimental groups were compared in vivo: defects filled with Tissucol-only constructs (fibrin, $n=10$ ) and defects filled with BMP-2 embedded in Tissucol constructs (fibrin-BMP, $n=8$ ). Both construct types contained $22.5 \mathrm{mg} / \mathrm{mL}$ fibrinogen. Constructs were implanted immediately after the defect creation. From both experimental groups, two animals were euthanised at 4 weeks (short follow-up) with an overdose of barbiturates (phenobarbital; $200 \mathrm{mg} / \mathrm{kg}$ body weight, TEVA Pharma, Haarlem, the Netherlands). The other animals were euthanised at 12 weeks (long followup). Of both short and long follow-up, bones and vessels surrounding them were analysed by $\mu \mathrm{CT}$ scanning and histology. The research protocol was approved by the animal experiments committee of the institution (104888-2/ 2014.III.06.054) and was in accordance with national law on animal experiments.

\section{Surgical procedure}

After at least $7 \mathrm{~d}$ of acclimatisation of the rats in the animal housing facility, a $6 \mathrm{~mm}$ critical-size segmental bone defect was created in the mid femur of all rats. Surgical procedures were performed aseptically under general anaesthesia (1-3.5 \% isoflurane). In short, the right hind leg was shaved from ankle to hip. The right femur was exposed through a lateral skin incision, dissection of soft tissue and 
division of underlying fascia. Using 3 proximal and 3 distal screws, a $23 \times 3 \times 2 \mathrm{~mm}$ polyether ether ketone plate was fixed to the anterolateral plane of the femur. Periosteum was removed over $6 \mathrm{~mm}$ of the mid-diaphyseal region before a $6 \mathrm{~mm}$ cortical bone segment was removed with a wire saw using a tailor-made saw guide (RISystem). After the construct (fibrin or fibrin-BMP) was press-fit implanted into the defect, the fascia and skin were sutured in layers using Vicryl rapide 5-0 (VR 2297; Ethicon). Subcutaneous injection of pain medication (buprenorphine, $0.05 \mathrm{mg} / \mathrm{kg}$ body weight) was given pre-operatively and twice a day for the following $3 \mathrm{~d}$, at which time the animals were also checked for any complications or side effects, such as wound problems and immobilisation of the operated leg, or any other abnormal behaviour.

\section{$\mu$ CT scanning}

Femora $\mu \mathrm{CT}$ scans were performed to assess bone formation at 4, 8 and 12 weeks. At baseline, a $\mu \mathrm{CT}$ scan was made directly after implantation of the construct, when rats were still under anaesthesia, to check for any complications. In supine position, the hind leg of the rat was fixated allowing scanning of the femur (scan time: $3 \mathrm{~min}$; voxel size: $42 \mu \mathrm{m}^{3}$; X-ray energy and X-ray tube potential: $90 \mathrm{keV}$; X-ray intensity: $180 \mu \mathrm{A}$; Quantum FX, PerkinElmer). Two regions of interest (ROIs) of $6 \times 3.5 \times 3.5 \mathrm{~mm}$ (bone volume inside the defect; BVi) and of $6 \times 6.4 \times 6.4 \mathrm{~mm}$ (total bone volume; TBV) were selected (Fig. 1). ROIs were segmented with a global threshold. Bone volume was measured in $\mathrm{mm}^{3}$ and three-dimensional (3D) reconstructions of the defects were made using image processing software (ImageJ, Java).

Bone bridging was assessed on ex vivo scans using ImageJ and was quantified by measuring the shortest remaining gap size between bone formed at the proximal and distal side of the $6 \mathrm{~mm}$ defect.

Vascularisation was examined at 4 weeks (short follow-up group, $n=2$ per group) and at end point of 12 weeks (all remaining animals, $n=8$ for the fibrin group and $n=6$ for the fibrin-BMP group) with the use of $\mu \mathrm{CT}$ angiography. Under general anaesthesia (1-3.5\% isoflurane in oxygen), a catheter was placed in the aorta abdominalis. First, $50 \mathrm{~mL}$ of $0.9 \%$ saline was injected, then $100 \mathrm{~mL}$ of $0.4 \%$ papaverine solution (P3510; Sigma-Aldrich), followed by $50 \mathrm{~mL} 0.9 \%$ saline and $100 \mathrm{~mL} 10 \%$ formalin. After injection of a lead chromate radiopaque contrast agent (Microfil MV-120, Flow Tech, Carver, MA, USA), the compound was polymerised overnight at $4{ }^{\circ} \mathrm{C}$. The next day, femora were removed en bloc and immersed in $4 \%$ phosphate-buffered paraformaldehyde for a week (Duvall et al., 2004). After decalcification, the volume of the polymerised vessels was measured by $\mu \mathrm{CT}$, in the same way as was done for bones.

\section{Histology}

All samples were retrieved for histology processing. After fixation, they were decalcified in a $10 \%$ ethylenediaminetetraacetic acid (EDTA)-PBS solution ( $\mathrm{pH} 7.4)$ and dehydrated in graded ethanol series (70-100 \%) and xylene. Subsequently, dehydrated samples were embedded in paraffin-wax and sectioned into $5 \mu \mathrm{m}$-thick slices (Microm HM340E; Thermo Fischer Scientific). Before each staining, samples were deparaffinised with xylene and gradually rehydrated through ethanol solutions (100$70 \%)$. Overall appearance and new bone formation were evaluated using H\&E staining (Sigma-Aldrich). CD34 staining was performed to identify endothelial cells and vessel-structures. Briefly, after blocking endogenous peroxidase activity for $10 \mathrm{~min}$ in $0.3 \%$ $\mathrm{H}_{2} \mathrm{O}_{2}$, proteolysis-mediated antigen retrieval was performed through $30 \mathrm{~min}$ incubation at $37^{\circ} \mathrm{C}$ with $0.1 \%$ trypsin (Sigma-Aldrich). After blocking with $5 \%$ bovine serum albumin (BSA)-PBS for $30 \mathrm{~min}$ at room temperature, CD34 antibody (1 : 200, AF4117; R\&D Systems) was incubated with $10 \%$ normal goat serum for $1 \mathrm{~h}$ at room temperature. After the washing steps, all samples were incubated in $1 \%$ BSA-PBS with an horseradish peroxidase (HRP)-labelled secondary antibody (1 : 200; P0449; Dako) for $30 \mathrm{~min}$ at room temperature. The labelling was visualised with diaminobenzidine as substrate. Then, sections were counterstained with haematoxylin, washed, dehydrated and mounted in DEPEX (Sigma-Aldrich). All sections were visualised using an Olympus BX51 microscope (Olympus DP70 camera). Histology merely served to confirm that mineralised tissue in the $\mu \mathrm{CT}$ represented indeed bone formation and to assess other types of tissue present. Therefore, histological results were not further quantified and scored.

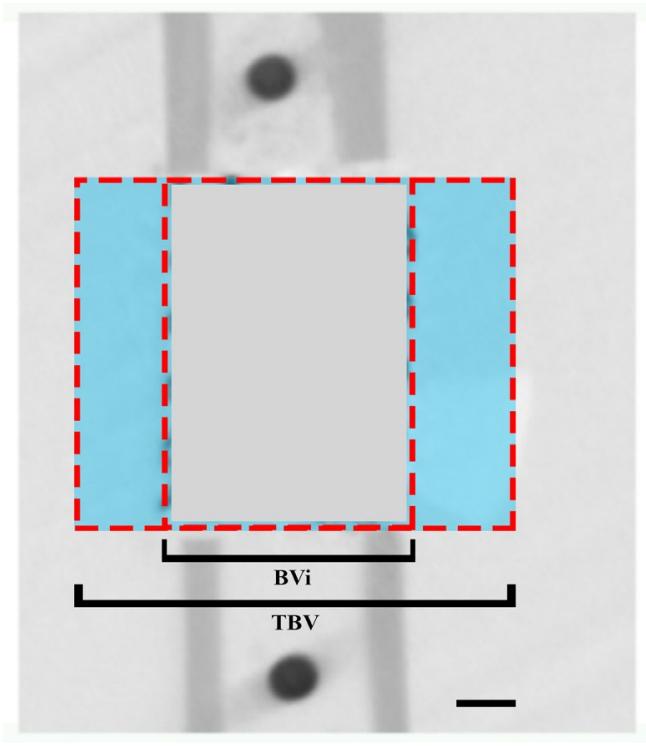

Fig. 1. ROIs around the defect area on a $\mu \mathrm{CT}$ scan of the rat femur. Sagittal view of a $\mu C T$ scan with two rectangular ROIs in red. The smaller red dotted box embracing the grey area indicates the ROI where BVi was determined. The outer red dotted box, embracing both the grey and blue area, shows the ROI where TBV was assessed. Scale bar: $1 \mathrm{~mm}$. 


\section{Statistics}

Statistical analysis was performed using SPSS Statistics 20.0 and 22.0 (SPSS, Inc.). Data are presented as means \pm standard deviation (SD). In the analysis of the in vitro release, mixed model analyses were used to test for statistical differences between the two groups (fibrin-BMP and fibrin-BMP-plasmin), with random intercept and corrected for time. For ALP activity, differences were tested with a oneway ANOVA for parametrical data and a KruskalWallis test for non-parametrical data. In the analysis of the results of the $\mu \mathrm{CT}$ scanning, mixed model analysis was used to test for statistical differences between the two groups (fibrin and fibrin-BMP), with random intercept and corrected for time. Gap size and vascularisation differences were tested with independent samples $t$-tests for parametrical data. If gap size or vascularisation were non-parametrical, Mann-Whitney U tests were performed. A $p<0.05$ was considered a statistically significant difference. A power calculation ( $\beta$-value $>0.80, \mathrm{SD} \sim 50 \%$ ) was made to find a true difference of at least a duplication in BVi and these values were based on previous work (Chatterjea et al., 2014; van der Stok et al., 2013a; van der Stok et al., 2013b; van der Stok et al., 2015). Based on this calculation, $n=5$ was required. The final decision to use more rats per groups accounted for animal loss (e.g. anaesthesia or defect-fixation failure).

\section{Results}

\section{In vitro experiment}

Cumulative BMP-2 release (Fig. 2a) showed that no burst release occurred within the first day for both the fibrin-BMP group and the fibrin-BMP-plasmin group. The first day, fibrin-BMP constructs released no BMP, whereas this was 54.48 ng from fibrin-BMPplasmin constructs. Both groups showed a more or less linear release profile that lasted until day 21 for the fibrin-BMP-plasmin group, with a cumulative release of $2.49 \pm 0.56 \mu \mathrm{g}$. In the fibrin-BMP group, the release continued linearly until the endpoint of $28 \mathrm{~d}$, reaching a maximum cumulative release of $0.73 \pm 0.46 \mu \mathrm{g}$. Already from day 5, BMP release in the fibrin-BMP-plasmin group was significantly increased relative to the group without plasmin, indicating an increased fibrin degradation by the plasmin. An average significant difference of $0.93 \mu \mathrm{g}$ was measured between the groups over time
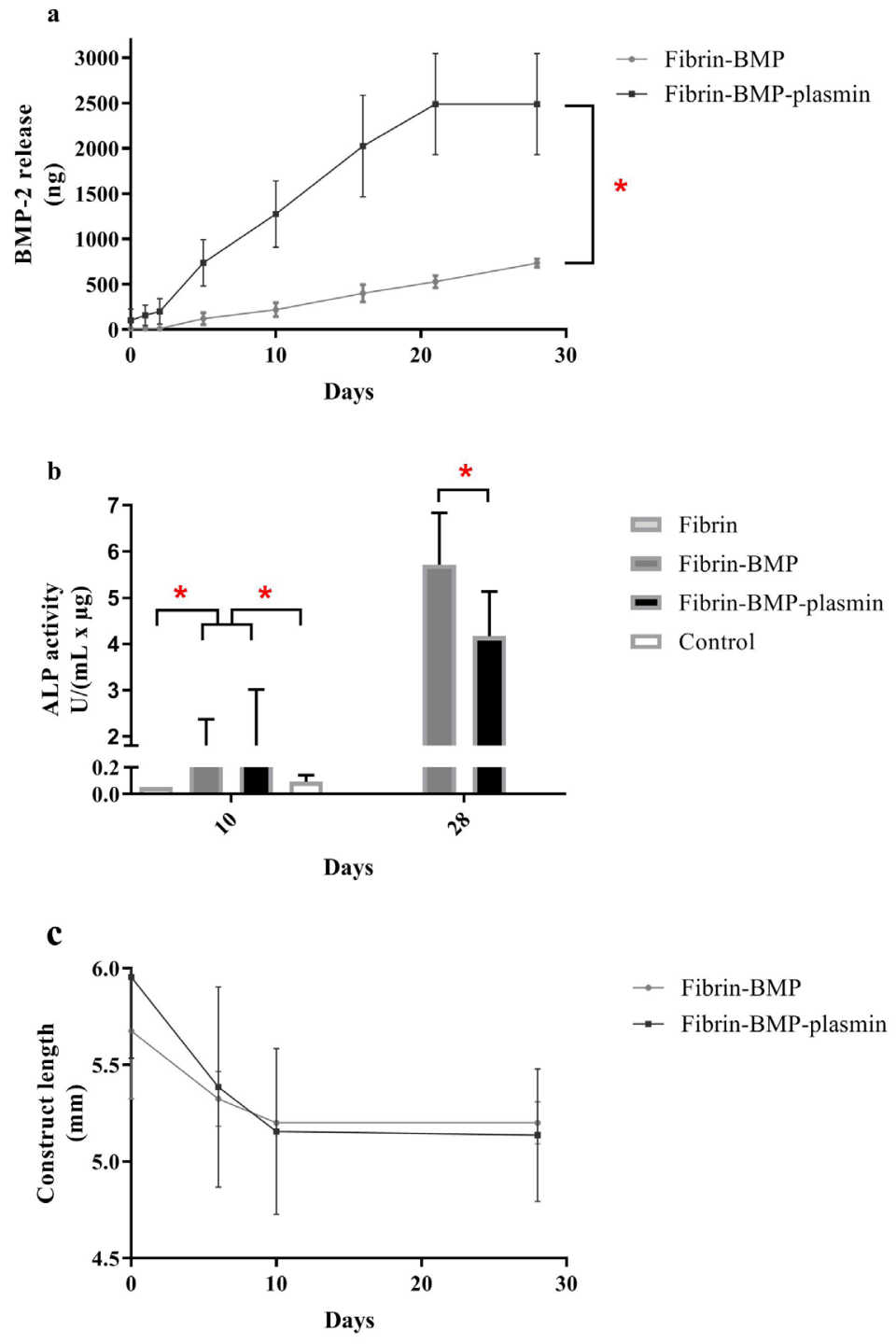

Fig. 2. Effects of in vitro incubation time and plasmin on BMP-2 release, activity and construct length. (a) In vitro BMP-2 release over time, (b) ALP activity at 10 and $28 \mathrm{~d}$ and (c) fibrin constructs length over time. ${ }^{*} p<0.05$. 
[confidence interval (CI) 0.41 vs. $1.45, p=0.008$ ]. For both groups, fibrin-BMP and fibrin-BMP-plasmin, the released BMP-2 at $10 \mathrm{~d}$ showed ALP activity (Fig. $2 b)$. At $28 \mathrm{~d}$, the fibrin-BMP group was slightly more active than the fibrin-BMP-plasmin group. Checking the construct length over time, no differences were observed when plasmin was added to the culture medium (Fig. 2c).

\section{In vivo experiment}

All rats were able to tolerate weight-bearing activities immediately after surgery; the implantation sites healed without complications and all animals remained healthy during the follow-up. Animals had an average weight of $414 \pm 21 \mathrm{~g}$ at time of implantation, with an increase of $36 \pm 10 \mathrm{~g}$ after 4 weeks and $73 \pm 11 \mathrm{~g}$ after 12 weeks.

\section{$\mu \mathrm{CT}$ analysis}

A significant difference was observed between the animal groups (fibrin and fibrin-BMP) with a mean difference over time of $38.2 \mathrm{~mm}^{3}$ (CI 29.8 vs. 46.6, $p<0.001$ ) for BVi and $46.4 \mathrm{~mm}^{3}$ (CI 34.6 vs. 58.2, $p<0.001$ ) for TBV (Fig. 3). At 4 weeks, fibrin showed $17.5 \pm 6.8 \mathrm{~mm}^{3} \mathrm{BVi}$ and $18.9 \pm 7.3 \mathrm{~mm}^{3} \mathrm{TBV}$, which increased to $32.5 \pm 6.0 \mathrm{~mm}^{3} \mathrm{BVi}$ and $41.8 \pm 9.4 \mathrm{~mm}^{3} \mathrm{TBV}$ after 12 weeks. Considerably more bone was observed at 4 weeks in the fibrin-BMP group $\left(63.6 \pm 7.6 \mathrm{~mm}^{3} \mathrm{BVi}\right.$ and $\left.80.8 \pm 21.4 \mathrm{~mm}^{3} \mathrm{TBV}\right)$ and this remained almost the same after 12 weeks $\left(63.5 \pm 13.1 \mathrm{~mm}^{3} \mathrm{BVi}\right.$ and $\left.74.9 \pm 14.8 \mathrm{~mm}^{3} \mathrm{TBV}\right)$. Compared to an intact femur, the fibrin-BMP group showed $110 \%$ of BVi and $105 \%$ TBV at 12 weeks (data not shown).

Histological evaluation and $e x$ vivo $\mu \mathrm{CT}$ analysis After 4 weeks of implantation, histology showed that tissue remodelling and endochondral bone regeneration were evident at the bone edges of the original defect in both the fibrin-BMP and fibrin group (Fig. 4a,b). Endochondral ossification fronts progressed towards the defect centre that was filled with vascularised fibrous tissue (Fig. $4 \mathbf{m}, \mathbf{n}$ ). New bone formation was characterised by the presence of hypertrophic chondrocytes (Fig. 4e,f) and formation of trabecular bone struts where cells resided in large lacunae (Fig. 4i,j). Among the trabeculae, haematopoietic bone marrow was found.

After 12 weeks, the progression of bone formation in the fibrin group had stopped (Fig. 4c) and the newly formed bone had a more mature appearance as compared to week 4 . Osteocytes at the bone edge in the original defect area resided in more flattened lacunae and bone had a lamellar appearance (Fig. $4 \mathbf{g}$ ). The defect was not bridged and fibrous tissue remained in the interspace (Fig. 4k). In contrast, the progression of the endochondral ossification fronts had continued until bridging of the defect had occurred for the fibrin-BMP group (Fig. 4d). Most of the cartilage remnants had disappeared by week 12 and more mature lamellar bone tissue was formed with osteocytes that resided in ellipsoidal lacunae (Fig. 4h). Also, haematopoietic bone marrow could be found in the cavities (Fig. 4l).

$\mu \mathrm{CT}$ scans over time showed most bone formation at the proximal side of the defects in the fibrin group, which was corroborated by the bone formation observed on histological slides (Fig. 5). Fibrin-grafted defects showed gradually increasing bone formation (Fig. 5a-c). In fibrin-BMP-grafted defects, most unorganised bone formation was before 4 weeks, with remodelling starting thereafter (Fig. 5d-f). Almost all these defects were bridged after 8 weeks, with the formation of an intramedullary channel, and almost complete remodelling was observed at 12 weeks after implantation. At 12 weeks, bone bridging was only seen in the cortical defects grafted with fibrin-BMP (Fig. 6). Only one of the defects of the fibrin-BMP group was not completely bridged and the average remaining gap size was $0.06 \pm 0.15 \mathrm{~mm}^{3}$, which was hugely different from the gap size in the fibrin group $\left(0.78 \pm 0.26 \mathrm{~mm}^{3}, p<0.001\right)$ (Fig. 6i).

Vessel volume was not different between fibrin-BMP and fibrin after $4\left(1.73 \pm 0.94 \mathrm{~mm}^{3}\right.$ and $0.96 \pm 0.68 \mathrm{~mm}^{3}$, respectively, data not shown) and 12 weeks $\left(0.71 \pm 0.32 \mathrm{~mm}^{3}\right.$ and $1.03 \pm 0.62 \mathrm{~mm}^{3}$, respectively) (Fig. 7), also not upon visual inspection (Fig. 6). CD34 staining was visible after 4 weeks in both groups and more CD34 staining was visible
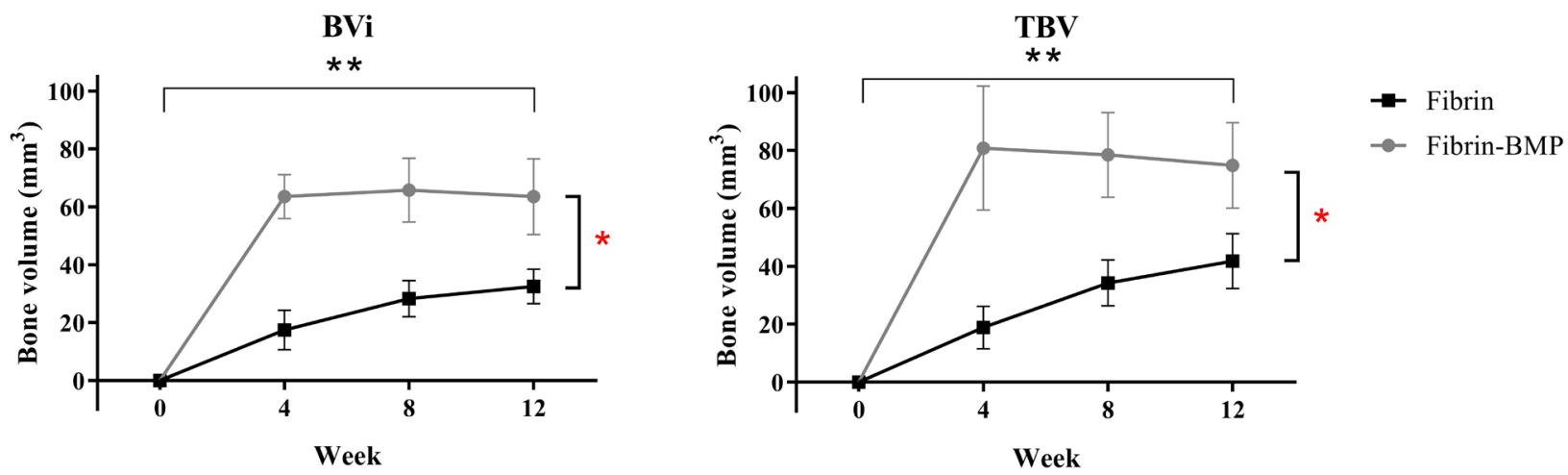

Fig. 3. Longitudinal quantification of bone regeneration in the segmental bone defects. In vivo $\mu \mathrm{CT}$ scan analysis after 4,8 and 12 weeks. Values are expressed as mean $\pm \mathrm{SD}(n=8$ for fibrin, $n=6$ for fibrin-BMP). ${ }^{*} p<0.05$ between groups, ${ }^{* *} p<0.05$ over time. 
inside the defects after 12 weeks in fibrin-grafted defects, indicating vessel formation (Fig. 7). This could be contributing to the relatively higher vessel density in the fibrous and muscle tissue infiltrating the defect, compared to the vessel density in the newly formed bone tissue.

\section{Discussion}

Commercially available fibrin loaded with rhBMP-2 was superior to fibrin controls, as evidenced by radiographic and histological bridging and bone volume as measured by $\mu \mathrm{CT}$ in a rat critical-size bone defect. In vitro results showed a slow release of rhBMP-2 from fibrin constructs in a controlled acellular in vitro environment. rhBMP-2 remained active after $28 \mathrm{~d}$ in this setting, as evidenced by ALP activity in ATDC5 cells after culture in preconditioned medium with rhBMP-2 released from the constructs. Results show that diluted clinically applicable fibrin was an excellent carrier for BMP-2 delivery.

Degradation properties of the fibrin used showed a continuous in vitro release and activity until day 28 without a burst release. This release pattern is different from that reported in other studies on the release kinetics of BMPs from fibrin (Li et al., 2016; Yang et al., 2010). In these studies, it was hypothesised that due to the relatively small size of BMPs as compared to the pores of fibrin and to their low affinity to interact with fibrin, BMPs are released

\section{4 weeks}

\section{2 weeks}

Fibrin
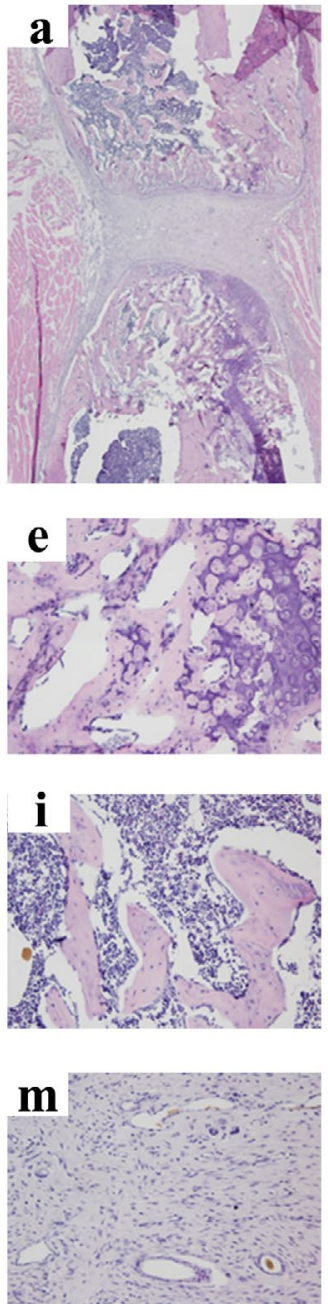

FibrinBMP
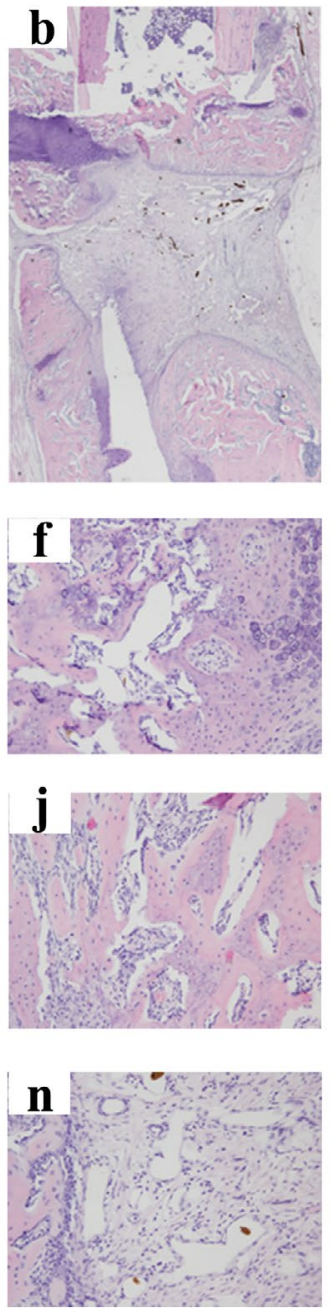

Fibrin
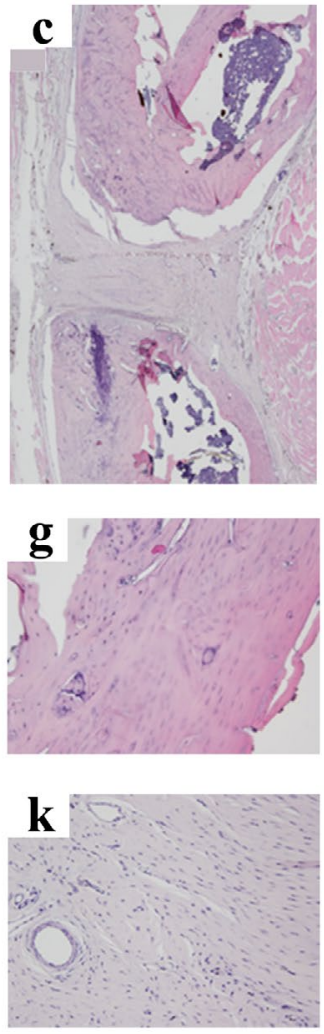

\section{Fibrin- BMP}
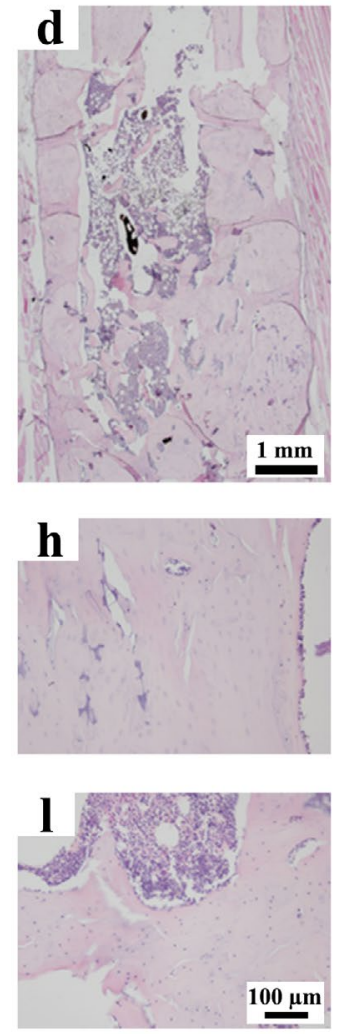

Fig. 4. Histological appearance (H\&E) of the defect area after 4 and 12 weeks in BMP-fibrin and fibrin control groups. (a-d) Overviews of the complete defect area are shown. (e,f) Hypertrophic chondrocytes and active endochondral bone formation; $(\mathbf{i}, \mathbf{j})$ young woven bone with large lacunae in a trabecular structure and $(\mathbf{m}, \mathbf{n})$ vascularised fibrous tissue in the central defect area at 4 weeks. (g,h) More mature lamellar bone could be observed after 12 weeks. (k) Fibrous tissue persisted in the fibrin controls at 12 weeks, while (l) only new bone and bone marrow was found in the fibrin-BMP group. 
quickly from the fibrin glue (Schmoekel et al., 2004). However, a much lower concentration of BMP was used in the present study and, therefore, BMP might be covalently crosslinked within the fibrin network structure by factor XIII (el-Hakim, 1999). However, more factors are involved in release and activity kinetics, such as the type of fibrin sealant (Noori et al., 2017). Most studies on this topic state that gradual release of BMP is preferred over a burst release, where most BMPs would get lost without becoming functional in vivo (Jeon et al., 2008; Schmoekel et al., 2005). The present study also seemed to indicate that, if the in vitro release profile is indicative for the in vivo release of $\mathrm{BMP}-2$, a gradual release of $\mathrm{BMP}-2$ is preferred.

Because plasmin and/or other factors might enhance degradation in vivo, fibrin degradation was also evaluated in vitro in the presence of plasmin.
Although macroscopically a decrease in the size of the fibrin constructs in the presence of plasmin was not observed, a faster BMP-2 release from these constructs was shown, likely related to the fact that plasmin made fibrin network more porous (Noori et al., 2017). A limitation of this method was its likely underestimation of the in vivo fibrin degradation and release profile. This could have contributed to the lack of any other enzyme or cellular action that may further affect BMP-2 release, as these are likely present in the in vivo situation. Indeed, the degradative process was further enhanced when the constructs were implanted in the rats, which was confirmed by the fact that fibrin was not retrievable on histology in constructs harvested 4 weeks after implantation.

Different types of fibrin sealants exist, with different biological, mechanical and degradation properties

\section{Time after implantation}

4 weeks

a

Fibrin

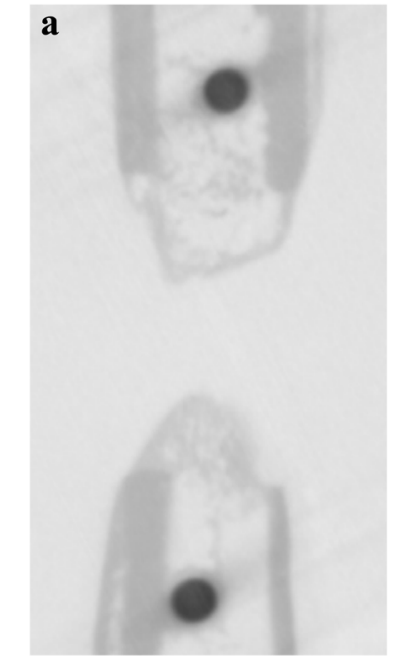

d

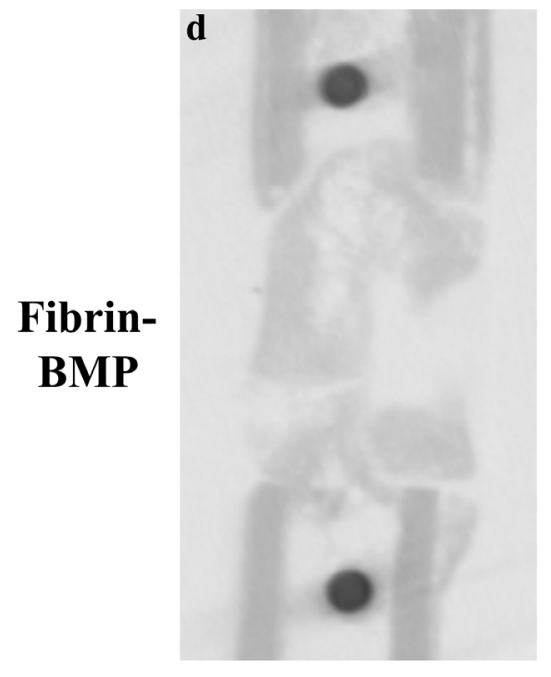
8 weeks

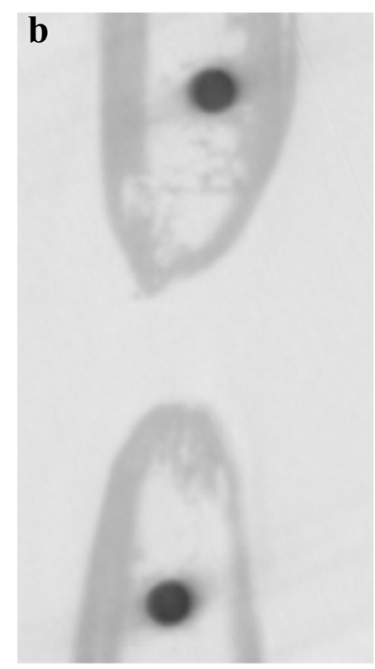

e

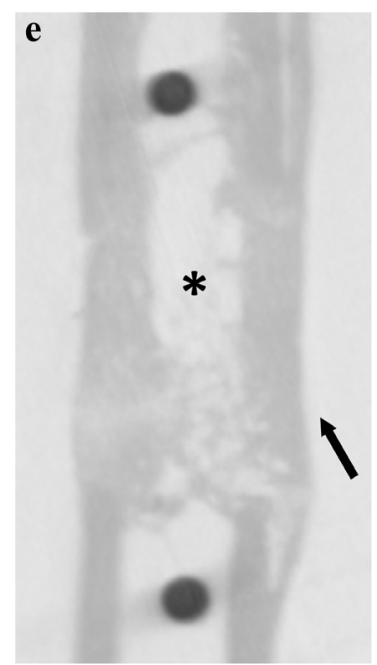

12 weeks

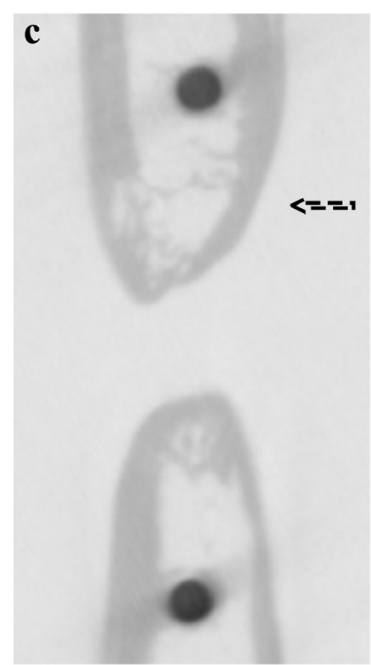

f

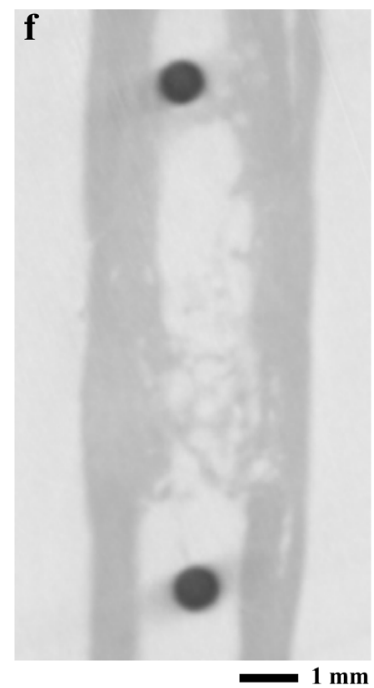

Fig. 5. $\mu \mathrm{CT}$ scans of segmental bone defects over time. Representative longitudinal $\mu \mathrm{CT}$ scans illustrating the bone regeneration process after grafting with $(\mathbf{a}, \mathbf{b}, \mathbf{c})$ fibrin or $(\mathbf{d}, \mathbf{e}, \mathbf{f})$ fibrin-BMP constructs. Asterisk shows formation of an intramedullary channel already at 8 weeks after implantation of fibrin with BMP-2. Solid arrow indicates a fully bridged defect. Dashed arrow shows bone formation from the proximal side of the femur in the fibrin-only-grafted defects. 
(Noori et al., 2017). The physicochemical properties, such as fibre thickness, porosity and permeability, depend on the conditions of polymerisation, such as fibrinogen and thrombin concentrations and other parameters, and are adaptable (Bluteau et al., 2006; Brown and Barker 2014; Davis et al., 2011; Nair et al., 1986; Okada et al., 1985; Weisel, 2005). However, the ideal properties of fibrin in the current application are not known and might vary depending on site or size of the construct used. In the present study, fibrinogen was diluted as much as possible while it could still form a gelated stable construct. Dilution was preferred, as a lower fibrinogen concentration is associated with a higher permeability, which is favourable for rapid cell invasion, remodelling and replacement by the transplanted or host cells (Smoljanovic et al., 2010).

The osteoinductive properties of BMP-containing fibrin were effective in non-loaded bone defects.
However, use of these fibrin constructs in loadbearing defects requires stabilisation (e.g. by internal or external fixation methods), which is not provided by the fibrin construct. Therefore, the fibrin should be combined with another type of scaffold to provide mechanical stability, e.g. porous titanium or ceramic scaffolds (Bluteau et al., 2006; van der Stok et al., 2015). Nevertheless, the advantage of the fibrin approach chosen in the present study included the possibility of injecting it directly after mixing fibrin and thrombin. Thereby, bone defects that are considered stable but still need stimulation of bone regeneration (e.g. delayed or non-unions) could be treated by percutaneous injection. Furthermore, fibrin is already FDA-approved and widely available.

Another important issue is the use of BMP-2 for clinical applications. Despite the significant evidence of BMP-2 potential for bone healing established using animal models, results are difficult to translate to

\section{$3 D \mu C T$ scans}

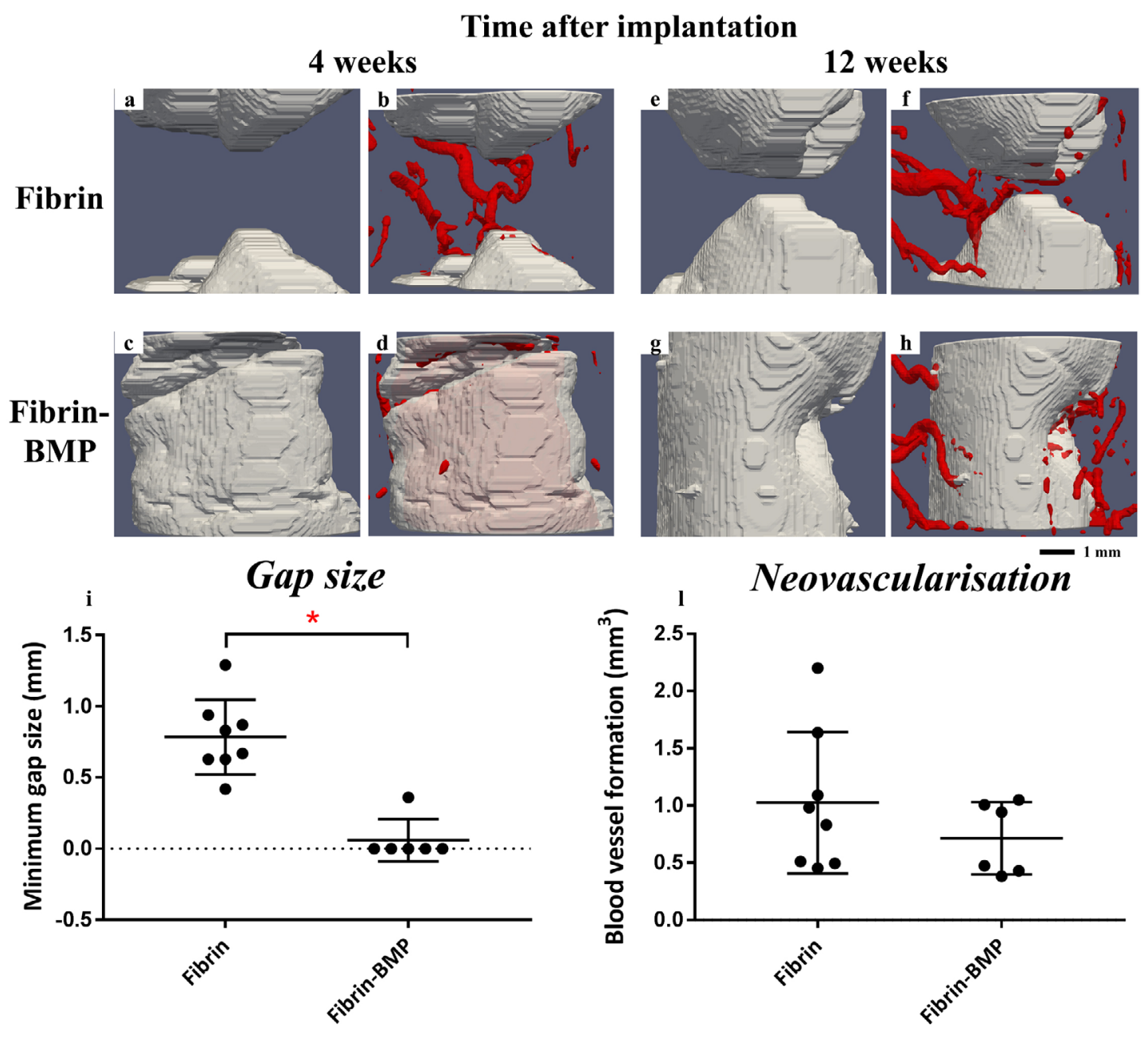

Fig. 6. 3D $\mu \mathrm{CT}$ renderings showing bone bridging and blood vessels around the bone with quantifications in the graphs below. Representative 3D $\mu \mathrm{CT}$ images of bone bridging and blood vessels after grafting with fibrin or fibrin-BMP constructs at (a-d) 4 weeks and (e-h) 12 weeks. (a,c,e,g) 3D reconstructions just before euthanasia. Bone appears in grey. $(\mathbf{b}, \mathbf{d}, \mathbf{f}, \mathbf{h}) 3 \mathrm{D}$ reconstructions just after euthanasia when Microfil had been injected into the blood vessels around and in the defect site. Bone appears in grey, blood vessels in red. (i) Remaining gap size indicated the bridging success. (1) Blood vessel formation after 12 weeks, measured by Microfil volume, represented neovascularisation around the bone. ${ }^{*} p<0.05$. 
humans (Zara et al., 2011). In humans, a much higher dose of BMP-2 seems to be necessary to generate bone, which might also elicit the reported negative side effects (Begam et al., 2017; Valentin-Opran et al., 2002). At this moment, BMP-2 use is FDA approved when applied using a collagen sponge as delivery vehicle. This delivery system could well be the reason for these side effects and the fact that high dosages of BMPs are necessary to achieve bone regeneration. Previously conducted experiments with a slowrelease collagen-based delivery system show less bone formation in the same bone defect model (van der Stok et al., 2013b) as compared to the results obtained in the present study and another study using a fibrinbased delivery system (van der Stok et al., 2015). Fibrin-based delivery systems might allow for the use of a much lower dose of BMP-2 due to its linear and long-term release profile. Schützenberger et al. (2012) also showed that a relatively low concentration of BMP-2 in fibrin carriers as compared to collagen carriers results in equivalent bone healing. However, based on the present study, it is not possible to draw the conclusion that fibrin was outperforming collagen as delivery vehicle for BMP-2, since collagen sponges with a similar dose of BMP-2 were not included. However, the results of the study indicated that using a commercially available fibrin gel to deliver BMP-2 was effective for enhancing bone regeneration in this rat bone defect model. The next step would be to compare the used construct, a commercially available fibrin gel containing BMP-2, to the commercially available collagen sponges containing BMP-2. Although, one could argue that it should be rather compared to the clinically used golden standard treatment, autologous bone. Nonetheless, this future experiment will likely contribute to the possible clinical application of BMP-2-loaded fibrin gels and, therefore, the use of a large animal model should be considered (e.g. goat or sheep) to encounter for the possibly negative effects of upscaling the scaffold volume for bone regeneration.

\section{Conclusions}

The study showed that treatment of a bone defect with BMP-2 embedded in diluted fibrin led to increased bone formation and even defect bridging and remodelling in a short time, without any ectopic bone formation. This suggested that fibrin might be an optimal carrier for BMP-2-induced bone regeneration.

\section{Acknowledgements}

Special thanks to Mattie van Rijen who assisted in the processing of the histology and Behdad Pouran who created the 3D images. This work was supported by the Netherlands Institute of Regenerative Medicine

\section{Time after implantation}

\section{4 weeks}
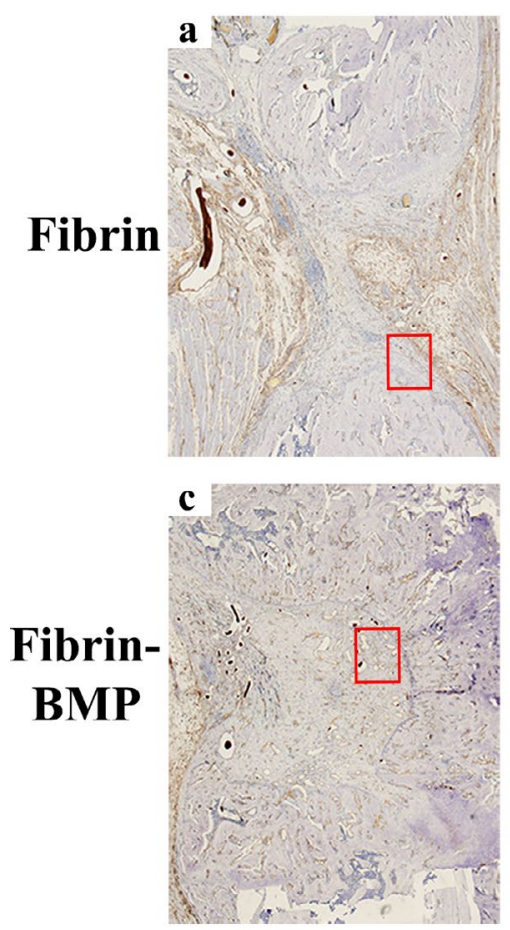
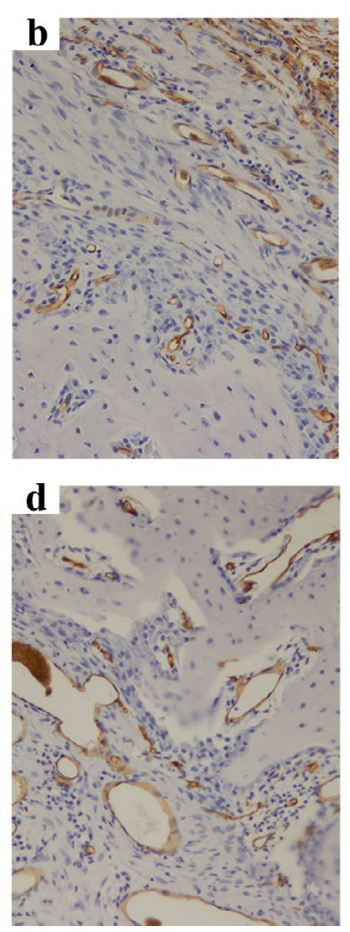

\section{2 weeks}
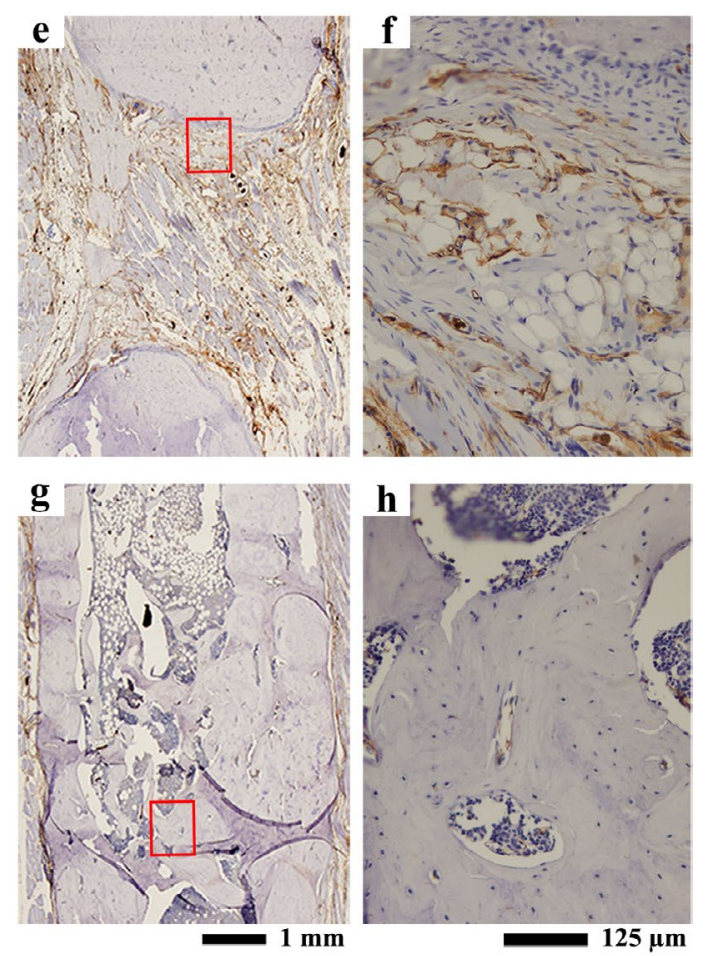

Fig 7. Histology of blood vessels in fibrin- and fibrin-BMP-grafted femora in rats. Representative images of CD34 staining taken from the centre of the defect after (a-d) 4 and (e-h) 12 weeks. Red boxes represent the areas that are shown in greater detail on the right-hand side in $\mathbf{b}, \mathbf{d}, \mathbf{f}, \mathbf{h}$. 
(grant number FES0908). Funding sources had no role in study design, collection, analysis and interpretation of data, writing of the manuscript nor decision to submit the article for publication.

\section{References}

Angle SR, Sena K, Sumner DR, Virkus WW, Virdi AS (2012) Healing of rat femoral segmental defect with bone morphogenetic protein-2: a dose response study. J Musculoskelet Neuronal Interact 12: 28-37.

Bannish BE, Chernysh IN, Keener JP, Fogelson AL, Weisel JW (2017) Molecular and physical mechanisms of fibrinolysis and thrombolysis from mathematical modeling and experiments. Sci Rep 7: 6914. DOI: 10.1038/s41598-017-06383-w.

Begam H, Nandi SK, Kundu B, Chanda A (2017) Strategies for delivering bone morphogenetic protein for bone healing. Mater Sci Eng C Mater Biol Appl 70: 856-869.

Benglis D, Wang MY and Levi AD (2008) A comprehensive review of the safety profile of bone morphogenetic protein in spine surgery. Neurosurgery 62: ONS423-431.

Bessa PC, Balmayor ER, Hartinger J, Zanoni G, Dopler D, Meinl A, Banerjee A, Casal M, Redl H, Reis RL, van Griensven M (2010) Silk fibroin microparticles as carriers for delivery of human recombinant bone morphogenetic protein-2: in vitro and in vivo bioactivity. Tissue Eng Part C Methods 16: 937-945.

Bluteau G, Pilet P, Bourges X, Bilban M, Spaethe R, Daculsi G, Guicheux J (2006) The modulation of gene expression in osteoblasts by thrombin coated on biphasic calcium phosphate ceramic. Biomaterials 27: 2934-2943.

Boerckel JD, Kolambkar YM, Dupont KM, Uhrig BA, Phelps EA, Stevens HY, Garcia AJ, Guldberg RE (2011) Effects of protein dose and delivery system on BMP-mediated bone regeneration. Biomaterials 32: 5241-5251.

Brown AC, Barker TH (2014) Fibrin-based biomaterials: modulation of macroscopic properties through rational design at the molecular level. Acta Biomater 10: 1502-1514.

Carragee EJ, Hurwitz EL, Weiner BK (2011) A critical review of recombinant human bone morphogenetic protein-2 trials in spinal surgery: emerging safety concerns and lessons learned. Spine J 11: 471-491.

Chatterjea A, van der Stok J, Danoux CB, Yuan $\mathrm{H}$, Habibovic P, van Blitterswijk CA, Weinans $\mathrm{H}$, de Boer J (2014) Inflammatory response and bone healing capacity of two porous calcium phosphate ceramics in critical size cortical bone defects. J Biomed Mater Res A 102: 1399-1407.

Davis HE, Miller SL, Case EM, Leach JK (2011) Supplementation of fibrin gels with sodium chloride enhances physical properties and ensuing osteogenic response. Acta Biomater 7: 691-699.
Dimitriou R, Mataliotakis GI, Angoules AG, Kanakaris NK, Giannoudis PV (2011) Complications following autologous bone graft harvesting from the iliac crest and using the RIA: a systematic review. Injury 42 Suppl 2: S3-15.

Dimitriou R, Tsiridis E, Giannoudis PV (2005) Current concepts of molecular aspects of bone healing. Injury 36: 1392-1404.

Duvall CL, Taylor WR, Weiss D, Guldberg RE (2004) Quantitative microcomputed tomography analysis of collateral vessel development after ischemic injury. Am J Physiol Heart Circ Physiol 287: H302-310.

Einhorn TA (1995) Enhancement of fracturehealing. J Bone Joint Surg Am 77: 940-956.

el-Hakim IE (1999) The effect of fibrin stabilizing factor (F.XIII) on healing of bone defects in normal and uncontrolled diabetic rats. Int J Oral Maxillofac Surg 28: 304-308.

Garrison KR, Donell S, Ryder J, Shemilt I, Mugford M, Harvey I, Song F (2007) Clinical effectiveness and cost-effectiveness of bone morphogenetic proteins in the non-healing of fractures and spinal fusion: a systematic review. Health Technol Assess 11: 1-150.

Giannoudis PV, Dinopoulos H, Tsiridis E (2005) Bone substitutes: an update. Injury 36 Suppl 3: S2027.

James AW, LaChaud G, Shen J, Asatrian G, Nguyen V, Zhang X, Ting K, Soo C (2016) A review of the clinical side effects of bone morphogenetic protein-2. Tissue Eng Part B Rev 22: 284-297.

Janmey PA, Winer JP, Weisel JW (2009) Fibrin gels and their clinical and bioengineering applications. J R Soc Interface 6: 1-10.

Jeon O, Song SJ, Yang HS, Bhang SH, Kang SW, Sung MA, Lee JH, Kim BS (2008) Long-term delivery enhances in vivo osteogenic efficacy of bone morphogenetic protein-2 compared to short-term delivery. Biochem Biophys Res Commun 369: 774780 .

Li Y, Li R, Hu J, Song D, Jiang X, Zhu S (2016) Recombinant human bone morphogenetic protein-2 suspended in fibrin glue enhances bone formation during distraction osteogenesis in rabbits. Arch Med Sci 12: 494-501.

Nair CH, Shah GA, Dhall DP (1986) Effect of temperature, $\mathrm{pH}$ and ionic strength and composition on fibrin network structure and its development. Thromb Res 42: 809-816.

Noori A, Ashrafi SJ, Vaez-Ghaemi R, HatamianZaremi A, Webster TJ (2017) A review of fibrin and fibrin composites for bone tissue engineering. Int J Nanomedicine 12: 4937-4961.

Okada M, Blomback B, Chang MD, Horowitz B (1985) Fibronectin and fibrin gel structure. J Biol Chem 260: 1811-1820.

Ong KL, Villarraga ML, Lau E, Carreon LY, Kurtz SM, Glassman SD (2010) Off-label use of bone morphogenetic proteins in the United States using administrative data. Spine (Phila Pa 1976) 35: 17941800. 
Rajangam T, An SS (2013) Fibrinogen and fibrin based micro and nano scaffolds incorporated with drugs, proteins, cells and genes for therapeutic biomedical applications. Int J Nanomedicine 8: 36413662.

Schmoekel H, Schense JC, Weber FE, Gratz KW, Gnagi D, Muller R, Hubbell JA (2004) Bone healing in the rat and dog with nonglycosylated BMP-2 demonstrating low solubility in fibrin matrices. J Orthop Res 22: 376-381.

Schmoekel HG, Weber FE, Schense JC, Gratz KW, Schawalder P, Hubbell JA (2005) Bone repair with a form of BMP-2 engineered for incorporation into fibrin cell ingrowth matrices. Biotechnol Bioeng 89: 253-262.

Schützenberger S, Schultz A, Hausner T, Hopf R, Zanoni G, Morton T, Kropik K, van Griensven M, Redl H (2012) The optimal carrier for BMP-2: a comparison of collagen versus fibrin matrix. Arch Orthop Trauma Surg 132: 1363-1370.

Smoljanovic T, Bicanic G, Bojanic I (2010) Update of comprehensive review of the safety profile of bone morphogenetic protein in spine surgery. Neurosurgery 66: E1030. DOI: 10.1227/ NEU.0B013E3181D8CCCD.

Termaat MF, Den Boer FC, Bakker FC, Patka P, Haarman HJ (2005) Bone morphogenetic proteins. Development and clinical efficacy in the treatment of fractures and bone defects. J Bone Joint Surg Am 87: 1367-1378.

Valentin-Opran A, Wozney J, Csimma C, Lilly L, Riedel GE (2002) Clinical evaluation of recombinant human bone morphogenetic protein-2. Clin Orthop Relat Res 110-120. DOI: 10.1097/00003086-20020200000011.

van der Stok J, Koolen MK, de Maat MP, Yavari SA, Alblas J, Patka P, Verhaar JA, van Lieshout EM, Zadpoor AA, Weinans H, Jahr H (2015) Full regeneration of segmental bone defects using porous titanium implants loaded with BMP-2 containing fibrin gels. Eur Cell Mater 29: 141-153.

van der Stok J, van der Jagt OP, Amin Yavari S, De Haas MF, Waarsing JH, Jahr H, Van Lieshout EM, Patka P, Verhaar JA, Zadpoor AA, Weinans H (2013a) Selective laser melting-produced porous titanium scaffolds regenerate bone in critical size cortical bone defects. J Orthop Res 31: 792-799.

van der Stok J, Wang H, Amin Yavari S, Siebelt M, Sandker M, Waarsing JH, Verhaar JA, Jahr H, Zadpoor AA, Leeuwenburgh SC, Weinans H (2013b) Enhanced bone regeneration of cortical segmental bone defects using porous titanium scaffolds incorporated with colloidal gelatin gels for time- and dose-controlled delivery of dual growth factors. Tissue Eng Part A 19: 2605-2614.

Wei G, Jin Q, Giannobile WV, Ma PX (2007) The enhancement of osteogenesis by nano-fibrous scaffolds incorporating rhBMP-7 nanospheres. Biomaterials 28: 2087-2096.
Weisel JW (2005) Fibrinogen and fibrin. Adv Protein Chem 70: 247-299.

Yang HS, La WG, Bhang SH, Jeon JY, Lee JH, Kim BS (2010) Heparin-conjugated fibrin as an injectable system for sustained delivery of bone morphogenetic protein-2. Tissue Eng Part A 16: 1225-1233.

Zara JN, Siu RK, Zhang X, Shen J, Ngo R, Lee M, Li W, Chiang M, Chung J, Kwak J, Wu BM, Ting K, Soo C (2011) High doses of bone morphogenetic protein 2 induce structurally abnormal bone and inflammation in vivo. Tissue Eng Part A 17: 1389-1399.

\section{Discussion with Reviewers}

Rodolfo De La Vega: There have been previous reports of fibrin used as a carrier for rhBMP-2. Why do you believe this has not moved forward into the clinic?

Authors: This could be due to the fact that during the last decade there has been a disrepute towards BMP-2 use, due to the reported negative side effects (Begam et al., 2017; Valentin-Opran et al., 2002).

Martijn van Griensven: How would you define the optimal BMP-2 content for patients?

Authors: The optimal BMP-2 content for patients is hard to predict, as there are multiple factors decisive in bone regeneration outcome. Such content is higher in humans as compared to rats, but this information is based on one delivery system (Termaat et al., 2005). It might be possible that a lower concentration is needed when a different carrier is used, as shown by Schützenberger et al. (2012).

Martijn van Griensven: Would you pre-prepare thrombin with BMP-2 for clinical usage or would you have the surgeons mix it in the operating room? Authors: We would prefer the surgeons to mix it, as it would mean less contamination, and, if possible, to use it as an injectable compound, which makes it easier to use in different locations and can be adjusted, if necessary.

Martijn van Griensven: Would other fibrin compounds be as good? E.g. autologous produced fibrin?

Authors: We cannot answer this question, as these types of fibrin were not compared. However, different types of fibrin (high molecular weight vs. mixed high and low molecular weight), which have been used in the present and another studies, perform both equally well (van der Stok et al., 2015). The question of performance remains if autologous-produced fibrin would be used. It may well be that they would perform equally well.

Editor's note: The Scientific Editor responsible for this paper was Chris Evans. 